\title{
Prediction of interfacial strength of HDPE overmolded with EPDM
}

\author{
W. Six, F. Desplentere \\ Materials Technology TC, KU Leuven, Bruges Campus, Belgium
}

G-J. Bex and A. Van Bael

Materials Technology TC, KU Leuven, Diepenbeek Campus, Belgium

\author{
J. De Keyzer \\ Sustainable Chemical Process Technology TC, KU Leuven, Diepenbeek Campus, Belgium
}

Abstract

Multicomponent injection molding often combines a stiff thermoplastic material with a thermoplastic elastomer or thermoplastic vulcanite. Recently, for high-demanding applications, a novel process has been developed to replace these thermoplastic elastomers or vulcanite with a thermoset rubber e.g. NBR or EPDM. One of the most important properties of a two component product is an adequate adhesion between both components. This paper describes a method which combines experiments and numerical simulations to predict the adhesion strength between a thermoplastic, semi-crystalline HDPE and an EPDM thermoset rubber. This method uses numerical simulations of the interfacial temperature and the local degree of cure. The local interface temperature is combined with the results of DSC measurements to predict the degree of melting, subsequently linked to the ability to develop adhesive strength. The degree of cure is used to determine the local rubber strength. The combination of the given data will be used to predict interfacial strength. The results obtained by proposed numerical strategy have been successfully validated with experimental data on a simple plate product.

1. Introduction

Multi-component injection molding is a common technique to produce complex parts which combine multiple polymeric materials. In this way, the final product benefits from the properties of both materials. The common method in this process is to first inject a thermoplastic polymer into an empty cavity and cool it down. Next, the cooled product is moved to a second, larger cavity and is overmolded with a second material [1]. Together, the two or more components form the finished product. This overmolding step is commonly done on the same machine in the same mold. In almost all cases the materials used in a multi-component product are a combination of thermoplastic and thermoplastic elastomeric (TPE's) materials. Less common is the combination of a thermoplastic material and a thermoset rubber.

In all cases, the most critical quality parameter is the adhesion between the different materials. For the most common situation, i.e. multi-component injection molding with two or more thermoplastic materials, various studies have been performed describing process settings and the influence of local parameters, e.g. temperature at the interface, on the resulting adhesive strength [2][3][4]. Three different mechanisms can occur at the interface in overmolded products: chemical bonding, chain entanglement and mechanical interlocking [5]. The latter is mainly due to product design and will not be discussed in this work. Chemical bonds and chain entanglement between two components are only possible when polymeric chain mobility is sufficiently high. This chain mobility is mainly determined by the local temperature [6]. Moreover, if the overmolded polymers can form a chemical reaction at the interface, e.g: hydrogen bonds, the resulting strength will be very high [6]. For various types of thermoplastics and elastomers or TPE's, research has been performed to estimate the adhesive strength as a function of process settings. Such research often includes an empirical design of experiments towards optimal 
processing parameters for a certain material combination. A. Islam et al, for example investigated adhesion in overmolding via this technique for 16 material combinations [7].

The development of adhesive strength on the interface in polymers is often referred to as healing. Various studies have been performed on the healing of amorphous polymer combinations. It is stated that healing of amorphous polymers can occur as soon as interfacial temperature exceeds the glass transition temperature. From this point, Brownian motions can occur and in that way polymer chains can interact and create entanglements across the interface [8] [9]. In optimal conditions, healing will increase gradually until the bond is as strong as the bulk polymer. This time to achieve complete healing is defined as the reptation time. It is stated that interfacial strength is at its maximum if molecular interdiffusion is equal or exceeds one radius of gyration [10].

For semi crystalline materials, healing can only start as soon as the material temperature exceeds the melting temperature. Levy et al [11] specified that the crystals present in the material prohibit the healing if the interface temperature is sub melting temperature. The study also states that reptation times are extremely short for semi crystalline materials and so full healing is found to be almost instantaneous. Once a semi-crystalline polymer is molten, healing of the material will be possible until temperature cools down below the crystallization temperature. The given method is frequently used to investigate and predict the interfacial strength between layers in thermoplastic composite materials. For example this theory is used to predict the local adhesive strength when an organosheet with certain semi crystalline matrix is overmolded with the same semi-crystalline polymer [11].

For the case where a thermoplastic material is overmolded by a thermoset rubber, only a few studies are available. Material suppliers of technical polymers have presented some essential data about material combinations that can achieve adhesion [12]. The presented combination criteria are mainly based on polarity and surface tension. They also state that the curing temperature of the thermoset rubber should be lower than the melting point of the thermoplastic material. With this statement they rule out the use of polyolefins for such applications, since their melting temperature is much lower compared to curing temperatures of the thermoset rubber. Bex et al [13][14], presented two studies on this topic, including mold design for a test product and a design of experiments (DOE) where technical polymers as well as polyolefins were combined with a thermoset rubber. From these design of experiments, including the presented HDPE-EPDM material combination, it was found that factors as rubber injection temperature, rubber injection speed, holding pressure and interface roughness have little to no influence on the final interfacial strength. The last parameter of this DOE, being the temperature between the components has found to be a very important parameter to have a good interfacial strength. This interfacial temperature is mainly determined by the mold cavity temperature. The optimal range for interfacial temperature is found to be just under the melting temperature for semi crystalline thermoplastic materials. For amorphous polymers, the optimal temperature has been found near the vicat softening temperature. Temperatures above melting cause large interfacial deformations and differential thermal contractions. This effect is similar as seen for thermoplastic overmolding situations. The modeling of interface healing is mainly done by the Genes model of polymer reptation [8]. This theory describes the mobility of polymer chains in a melt. This movement is restricted by neighboring and entangled polymer chains. In this theory, polymer chains are allowed to move back and forth within an idealized tube. This theory, based on probability of chains moving across the interface has been used by Prager et al [3] to model and predict the diffusion of polymer chains across polymer-polymer interfaces. If polymer chains start to cross the interface, the interfacial strength starts to increase which is known as the healing of the interfaces. The amount of healing is described as 'degree of healing', $\mathrm{D}_{\mathrm{h}}$. It can be determined by the fraction of the maximum attainable bond strength $\left(\sigma_{\infty}\right)$. [15]

$$
D_{h}=\frac{\sigma}{\sigma_{\infty}} \propto\left(\frac{t}{t_{r}}\right)^{\frac{1}{4}}
$$


The characteristic time to complete healing is given as the reptation time, also being the time that interdiffusion comes to an end . However, Wool [16], showed that the reptation theory is mainly valid for low molecular weight polymers where $M_{c}<M<8 M_{c}$, where $M_{c}$ is the critical entanglement molecular weight. For semi crystalline materials where the molecular weight is typically higher than $8 M_{C}$ the interfacial strength reaches its maximum before interfusion comes to an end, and thus for these polymers the reptation time $\left(\mathrm{t}_{\mathrm{r}}\right)$ provides a conservative prediction. The time needed to achieve complete healing in these type of polymer is defined as the welding time $t_{w}$. This welding time is defined by Yang and Pitchumani [17] via equation 2

$$
D_{h}(t)=\frac{\sigma(t)}{\sigma_{\infty}}=\left[\int_{0}^{t} \frac{1}{t_{w}(T(t))} d t\right]^{1 / 4}
$$

For these engineering plastics Yang and Pitchumani state that healing occurs above the melt temperature, so polymer chains are free to migrate. The welding time presented in this work for PEEK, (Polyetheretherketone) has a value of $0.11 \mathrm{~s}$ at a reference temperature of $400{ }^{\circ} \mathrm{C}$ and is influenced by temperature determined by an Arrhenius equation.

Bex et al. also state that curing should be above $90 \%$ upon ejection of the product to insure sufficient and completed chain entanglement between rubber and thermoplastic material. As a last important finding, they state that the order of injection is very important. The thermoplastic material should be injected first and then overmolded with the thermoset rubber. In that way, the uncured rubber creates entanglements with the thermoplastic polymer chains, which is impossible when processing is done in the opposite order. If the rubber is cured first, almost no chain mobility is left to heal towards the thermoplastic material. Although the presented data gives insight in the important parameters for the process, no in-depth study is available up to now to predict the interfacial strength as a function of process settings.

In order to investigate the injection molding process and the properties of the resulting product, numerical simulations are widely used and developers are still improving the software to have more accurate results. Also to predict interfacial strength in overmolded thermoplastic products advances have been made [18][19]. However, as far as known by the authors, no real numerical simulation tools are commercially available to predict the interfacial strength for the given material combinations. Moreover, In Autodesk Moldflow, for the thermoset rubber - thermoplastic overmolding situation, there is no predefined method to simulate this process. In the present study, this problem was addressed by using a reactive injection molding simulation in which the first component is included as a part insert. This method proved to be adequate for the given process [20].

Adhesion between two components can fail in three different manners. The failure is adhesive when the product fails exactly at the interface and none of the samples themselves fail, cohesive, when the substrate fails near the interface instead of the interface itself, or mixed, when failure is partially adhesive and partially cohesive. In many cases it is stated that a cohesive failure of the component is wanted. In that way, the bonding is at least as strong as the substrate, and further increase of the bonding strength has no use. However, in the presented study, the strength of the thermoset rubber, being one of the substrates, increases as the degree of cure increases. So, next to increasing the adhesive strength, also the cohesive strength of the rubber as a function of the curing time must be investigated.

\section{Mold and materials}

For the investigation of adhesive strength between several thermoset rubbers and thermoplastic materials, Bex et al [13] developed a mold to produce two-component injection molded plate products. Special attention was given to the thermal separation in the cavity of the mold, allowing higher mold surface temperatures for the rubber part. This is required to cure the thermoset rubber while the thermoplastic material remains sufficiently cooled. The complete plate product $(150 \times 80 \times 2 \mathrm{~mm})$ consists of 2 equally large thermoset rubber and thermoplastic parts having a width of $40 \mathrm{~mm}$. The sample production was performed on an ENGEL 100t ES330H 80V 80HL-F injection molding machine with vertical rubber 
injection unit (screw $\varnothing 25 \mathrm{~mm}, \mathrm{~L} / \mathrm{D} 16$ ) and horizontal thermoplastic injection unit (screw $\varnothing 30 \mathrm{~mm} \mathrm{~L} / \mathrm{D} 20$ ). The temperature of the rubber injection unit is externally controlled by a Tool Temp TT-157 E tempering device at $80^{\circ} \mathrm{C}$. The temperature of the mold was controlled by two HB THERM: HB-180Z2 and a HB THERM: HB180Z3 tempering unit, the latter is used if to produce the water at $180 \mathrm{C}$ at a pressure of 12 bars and is combined with one $\mathrm{HB}-180 \mathrm{Z2}$, set a $80 \mathrm{C}$ in a variotherm control system. By switching between $180^{\circ} \mathrm{C}$ and $80^{\circ} \mathrm{C}$, the curing time of the thermoset rubber can be controlled.

The production process is illustrated in Figure 1, left. In the first phase, the complete mold is set at a low specified mold temperature to produce the thermoplastic product. In this phase, multiple thermoplastic components are produced, and are allowed to cool down to room temperature. In the second molding step, the metal insert is removed from the mold and the mold temperature at the empty cavity is set at curing temperature, while the rest of the mold stays cooled. The latter temperature is not necessarily the same temperature as in the molding phase of the thermoplastic part. By varying the water temperature of the thermoplastic cavity in the second phase, the interface temperature between the thermoplastic part and thermoset rubber can be controlled, given that this temperature should not exceed a certain value to avoid excessive melting of the interface. The water temperate for curing, $\mathrm{T}_{\mathrm{m} \text { high, }}$ will be $180^{\circ} \mathrm{C}$ and is the same for all tests. After mold surface temperature stabilization, a molded thermoplastic part is inserted and is overmolded with the thermoset rubber. Between insertion of the thermoplastic and overmodling phase a waiting time of $120 \mathrm{~s}$ is added to the process after closing the mold to allow thermal stabilization of the thermoplastic part. Table 1 shows the general process parameters.

Table 1: General process parameters

\begin{tabular}{lrr}
\hline Process parameters & HDPE & EPDM \\
\hline Injection temperature $\left[{ }^{\circ} \mathrm{C}\right]$ & 230 & 80 \\
Mold temperature $\left[{ }^{\circ} \mathrm{C}\right]$ & 20 & 180 \\
Injection flow rate $\left[\mathrm{cm}^{3} / \mathrm{s}\right]$ & 40 & 60 \\
Holding pressrue $[\mathrm{bar}]$ & 790 & 200 \\
Holding time $[\mathrm{s}]$ & 15 & 200 \\
\hline
\end{tabular}



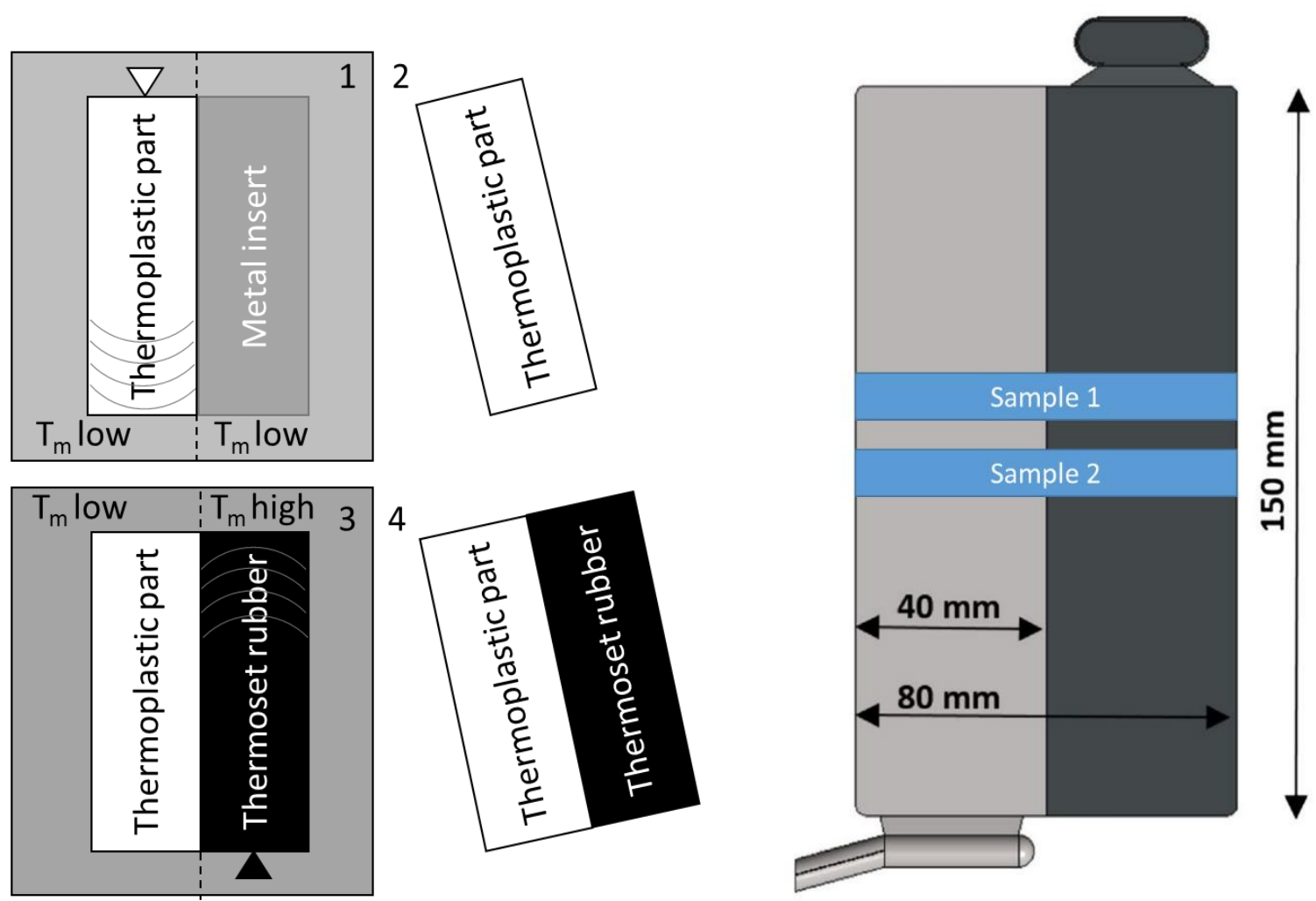

Figure 1: procedure for production of two component thermoplastic, thermoset rubber plate products[20](left). Location of sample taking in plate product, dimensions in mm (right) [13]

In the presented study, a high-density polyethylene, HDPE thermoplastic material being: Sabic HDPE M80064SE (with MFI 8g/10min) will be combined with a EPDM thermoset rubber, Hercorub EPDM 005. The compound formulation is given in Table 2: compound formulation for Hercorub 005KTable 2 in parts per hundred resin (phr)

Table 2: compound formulation for Hercorub $005 \mathrm{~K}$ in parts per hundred resin (phr)

\begin{tabular}{lr}
\hline Ingredient & $\boldsymbol{p h} \boldsymbol{r}$ \\
\hline Oil extended EPDM & 200 \\
Carbon black & 133 \\
Oil & 56 \\
Stearic acid & 1.1 \\
Zinc oxide & 5 \\
Accelerators & 5.2 \\
Sulfur & 1.2 \\
Retarder & 0.5 \\
\hline
\end{tabular}

Testing of the interfacial adhesive strength is done by tensile testing samples of the produced plate product. Two tensile strips are punched out of every plate. Sample dimensions are 80 x 10 x $2 \mathrm{~mm}$. The location where the sample is taken out of the product is shown in Figure 1, right. This location is important as the adhesive strength can be influenced by local dimensions of the thermoplastic part or by temperature differences in the mold. The final properties of the sample are tested with an Instron 3365 tensile testing machine, with a testing speed of $200 \mathrm{~mm} / \mathrm{min}$. 


\section{Investigation of influencing factors on adhesion}

In this section, the influencing factors that define the interfacial strength, in particular the interface temperature and degree of cure, will be investigated.

\subsection{Thermoplastic interface temperature.}

As stated in the introduction, healing for amorphous polymers starts as soon as the temperature exceeds $\mathrm{T}_{\mathrm{g}}$, for semi-crystalline materials healing is only possible in the melting state and is stated to be instantaneous [11] due to the high chain mobility in this melt phase. Instead of using a single melting temperature to model healing, a melting trajectory has proven to be more accurate[11]. The study presented by Bouwman et al [11] uses the melting behavior measured by Differential Scanning Calorimetry (DSC) to predict local interfacial strength. In this study, numerical simulations are used to predict the interfacial temperature and are combined with the results of DSC measurements to predict the degree of melting which is subsequently linked to the local degree of healing. The degree of melting is defined as the relative amount of material in the melting phase. The melting trajectory of the semicrystalline HDPE is shown in Figure 2, left. It was obtained experimentally by DSC analyses (TA Q200) for three heating rates, being 5,10 and $20^{\circ} \mathrm{C} / \mathrm{min}$, this to investigate the influence of this heating rate on the degree of melting. The base line is added to measure the area of the melting peak. The normalized, cumulative area of the melting peak of the HDPE M80064SE is shown in Figure 2, right for the three given heating rates and can be fitted by an exponential curve. The variation in degree of melting between the three heating rates is very limited if the temperature is below $130^{\circ} \mathrm{C}$ this corresponds with a degree of meting of 0.4. at higher temperatures, the difference in heating rate shows to have an influence on the degree of melting.
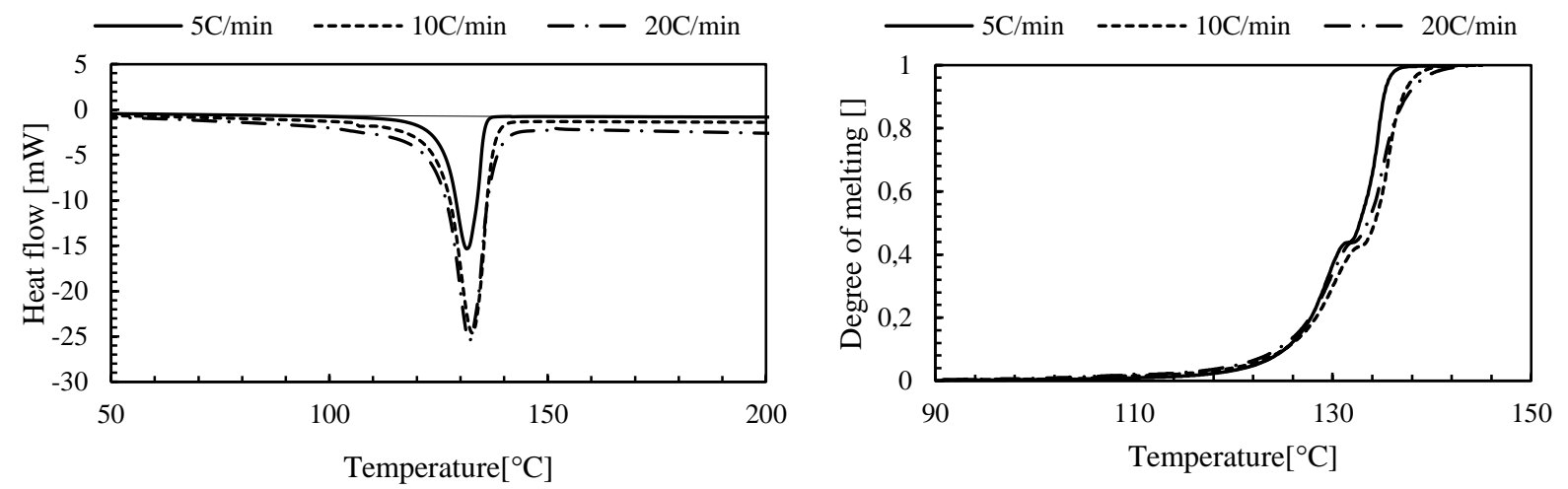

Figure 2: melting trajectory of SABIC HDPE M80064SE, heating rate of 5, 10 and 20K/min (left). Degree of melting $D_{m}$ as a function of temperature and heating rate for Sabic HDPE M80064SE (right)

Bouwman et al. [11] state that the degree of melting $\mathrm{D}_{\mathrm{m}}$ is directly correlated with the degree of healing. At a value for $D_{m}$ of zero, no healing is possible. Due to crystallites in the material, chain movement is then restricted. If melting starts, crystallites start to dissolve in the melting phase and chain mobility increases. At a value of one, no crystallites are left and complete healing is possible. The difference between the three heating rates is found to be very low at temperatures $<130^{\circ} \mathrm{C}$, or a $\mathrm{D}_{\mathrm{m}}$ of 0.4 .

Determination of the maximum adhesive strength versus the interface degree of melting can be done by tensile testing of samples produced with a predefined interfacial temperature. After testing, a pure adhesive failure is essential, otherwise, the given data cannot be used to determine the adhesive strength as a function of the degree of melting at the interface. To achieve this, complete curing of the thermoset rubber is critical. Figure 3 shows the result of this test for the given HDPE material, combined with the 
Hercorub EPDM 005K.The given graph shows an asymptotic regression function, defined by equation 3.

$$
\sigma_{D m}=\theta_{1}+\theta_{2}\left(1-e^{-\theta_{3} D_{m}}\right)
$$

The maximum adhesive strength is almost reached as soon as the degree of melting reaches a value of 0.4. Due to this reason, difference in degree of melting as a function of the heating rate can be neglected.

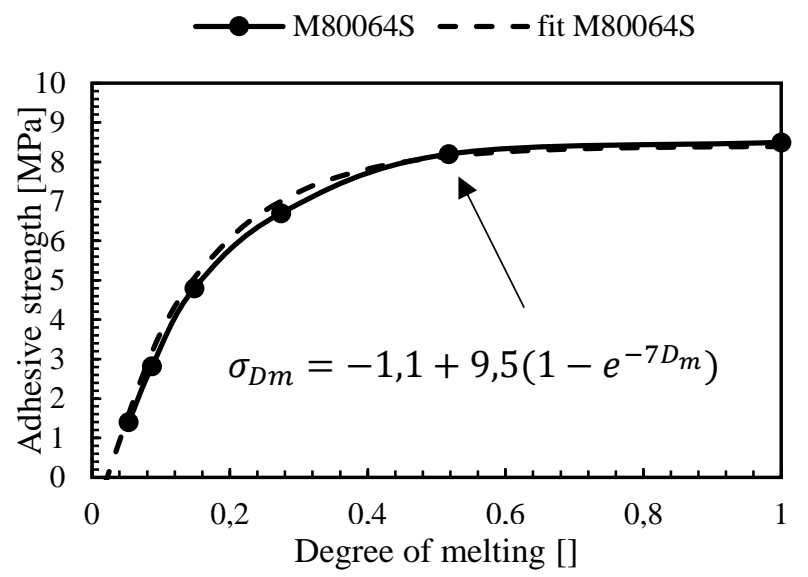

Figure 3: measured adhesive strength as a function of degree of melting of Hercorub EPDM 005K combined with Sabic HDPE M80064S

\subsection{Rubber curing degree.}

As stated by Bex et al [13], the rubber curing degree at the interface is the second important parameter for optimal adhesion since curing of the rubber reduces chain mobility. Due to low chain interactions, uncured material has a very low $(<0.25 \mathrm{MPa})$ stiffness and tensile strength. During the curing phase, sulfur bonds are created and chain mobility decreases drastically. A restricted chain mobility is of key importance to interlock the diffused thermoplastic chains. Predicting the degree of cure of the thermoset rubber is done through the Kamal model [21], given in equation 4. This is the standard curing model included in the simulation software Moldflow. In this equation, the values of $K_{1}$ and $K_{2}$ are given by equation 5 where $\alpha=$ the degree of cure, $T=$ the temperature and $t=$ time, $i=1,2$. The other variables, $\mathrm{A}_{\mathrm{i}}, \mathrm{E}_{\mathrm{i}}, \mathrm{m}$ and $\mathrm{n}$ are data fitted variables

$$
\begin{gathered}
\frac{d \alpha}{d t}=\left(K_{1}+K_{2} \cdot \alpha^{m}\right)(1-\alpha)^{n} \\
K_{i}=A_{i} \cdot \exp \left(-\frac{E_{i}}{T}\right)
\end{gathered}
$$

The values of the Kamal model can be found by data fitting via the mathematical Grey-Box Model Estimation [26] method on the curing data of the EPDM thermoset rubber. In the presented study, the data is determined by a Moving Die Rheometer (MDR) apparatus (MDR 2000 Alpha technologies) according to test standard ASTM D5289. The disadvantage of the Kamal model is the inability to predict de-vulcanization. Another model has been presented by Milani et al. [22] and Isayev et al. [23] including this phase. To test the de-vulcanization behavior of the material, an MDR test was performed at $180^{\circ} \mathrm{C}$ for 1800s. This test showed a de-vulcanization of $4 \%$. The energy of the exothermal reaction was determined by DSC and measured to be $0.6 \mathrm{~J} / \mathrm{g}$. As this value is very low only limited internal heating due to the curing can be expect.. The fitted values are given in Table 3 and are used for numerical simulations. 
Table 3. Parameters for the Kamal Model for Hercorub EPDM $005 \mathrm{~K}$.

\begin{tabular}{lrl}
\hline Parameter & Value & Unit \\
\hline $\mathrm{A} 1$ & 10000 & {$[1 / \mathrm{s}]$} \\
$\mathrm{A} 2$ & $2.38 \mathrm{E}+08[1 / \mathrm{s}]$ \\
$\mathrm{E} 1$ & $8608.2[\mathrm{~K}]$ \\
$\mathrm{E} 2$ & $10404.8[\mathrm{~K}]$ \\
$\mathrm{m}$ & 0.6[] \\
$\mathrm{n}$ & 1.249[] \\
\hline
\end{tabular}

The local rubber strength is linearly correlated with the degree of cure. The occurring rubber strength can be estimated by determination of the green rubber strength $\left(\sigma_{\alpha=0}\right)$ and full rubber strength $\left(\sigma_{\alpha=1}\right)$ and linear interpolation by means of equation 6 [24]. The green rubber strength, $\sigma_{\alpha=0}$ for the Hercorub EPDM 005K is measured to be $0.25 \mathrm{MPa}$, the ultimate rubber strength $\left(\sigma_{\alpha=1}\right)$ is $7.54 \mathrm{MPa}$

$$
\sigma_{\alpha}=\sigma_{\alpha=0}+\frac{\sigma_{\alpha=0}}{\sigma_{\alpha=1}} \alpha
$$

Curing of the thermoset rubber at $180^{\circ} \mathrm{C}\left(\mathrm{T}_{\mathrm{m}}\right.$ high) occurs in less than 5 minutes. In this way, one would estimate that full curing of the thermoset product would occur fast and always within reasonable processing times. However, at the interface, the temperature must be much lower in order to avoid excessive melting of the thermoplastic part. As a result, reaching a degree of cure of $90 \%$ at the interface can take up to 40 minutes at the melting temperature of the HDPE material (i.e. at $135^{\circ} \mathrm{C}$ ). If curing is incomplete, in case of lower cycle times, this will evidently affect the interfacial strength. Using a thermoplastic material with a higher melting temperature is mainly advised by material suppliers. This limits however the material selection for the customer.

\subsection{Prediction of the interfacial strength.}

The resulting interfacial strength depends on both the adhesive strength, or strength of the interface itself, and the cohesive strength, i.e. the strength of the materials near the interface. For these kind of two component products, adhesive strength is, as stated before, mainly determined by the interfacial temperature during curing, which in its turn is governed by the mold temperature. The degree of cure, determined by interfacial temperature and curing time, mainly determines the strength of the rubber material near the interface. The strength of the HDPE material is barely influenced by the interfacial temperature and is much higher compared to the maximal rubber strength. Determination of the local interface strength can be done by comparing the adhesive strength, defined by the simulated degree of healing, and the local rubber strength. By comparing these values, the local interface strength can be determined. This is given in equation 7 where $\sigma_{\alpha}$ defines the maximal rubber and $\sigma_{D m}$ defines the adhesive strength defined by the equation given in Figure 3

$$
\sigma_{I}=\left\{\begin{aligned}
\sigma_{\alpha}, & \sigma_{\alpha}<\sigma_{D m} \\
\sigma_{D m}, & \sigma_{\alpha} \geq \sigma_{D m}
\end{aligned}\right.
$$

\section{Simulations and results}

First the simulation model will be discussed, together with the results for temperature and curing. In the next section, the results will be transformed into local adhesive and cohesive strength, which will be combined in summary plots in section 5 showing the complete interface with local interface strength results.

\subsection{Simulation model}


Numerical simulations are used to assist in the prediction of the interfacial properties of the given two component product. For the given product type the reactive solver in Moldflow Insight 2018 is used, this solver can be used to simulate the filling and curing behavior of reactive materials in injection molding. For all situations a complete 'Cool(FEM) Fill Pack' simulation is performed. The given simulation type first calculates the mold temperature distribution. In the second phase, the software simulates the filling behavior of the cavity and finalizes the simulation by performing a packing. During the filling and packing the curing continues calculated. The mold and product are meshed with linear tetrahedron elements. For the product, 8 elements through thickness are used. The cooling channels are meshed with linear beam elements, with an L/D of 2. The HDPE component is inserted in the simulation as a 'part insert' . The properties of this part insert and the thermal properties of the mold are shown in table 2 . The viscosity curve of the injected rubber material has been measured by a dual bore rheometer. The reactive viscosity model [25] is fitted via nonlinear least square fitting on this data. The model is defined by Equation 8

$$
\eta(\alpha, T, \dot{\gamma})=\frac{\eta_{0}(T)}{1+\left(\frac{\eta_{0}(T) \cdot \dot{\gamma}}{\tau^{*}}\right)^{n-1}} \cdot\left(\frac{\alpha_{g}}{\alpha_{g}-\alpha}\right)^{\left(C_{1}+C_{2} \cdot \alpha\right)}
$$

where $\eta=$ viscosity, $\alpha=$ degree of cure, $\dot{\gamma}=$ shear rate, $T=$ temperature, $\tau^{*}=$ shear stress. The other parameters $C_{1}, C_{2}, \alpha_{g}$ and $n$ are extracted from the data fitting. The model is composed out of two factors. The first factor in equation 7 is the cross viscosity model, with exponential temperature dependency and as second term, the conversion model. The viscosity at a zero shear, $\eta_{0}$ is given by equation 9 the data fitted values are shown in table 3.

$$
\eta_{0}(T)=B \cdot \exp \left(\frac{T_{b}}{T}\right)
$$

Due to the measurement method, the viscosity could only be measured for uncured material, and in that way, the dependency of viscosity on the curing could not been determined. However, as the viscosity model is mainly used during the injection phase which lasts for less than $0.5 \mathrm{~s}$ and during which no curing occurs, this dependency is not required for accurate simulation results. As result, the parameters $\mathrm{C} 1$ and $\mathrm{C} 2$ are respectively set to 1 and 0 in the given viscosity model.

Table 4: properties of HDPE insert and mold

\begin{tabular}{lrrl}
\hline \multirow{2}{*}{ Parameter } & \multicolumn{1}{l}{ Value } & \multicolumn{1}{c}{ Unit } \\
\cline { 2 - 3 } & M80064SE & Mold: $\boldsymbol{P 2 0}$ & \\
\hline $\mathrm{Cp}$ & 2822 & 460 & {$[\mathrm{~J} / \mathrm{kgK}]$} \\
$\lambda$ & 0.26 & 29 & {$[\mathrm{~W} / \mathrm{mK}]$} \\
$\rho$ & 0.952 & 7.8 & {$\left[\mathrm{~g} / \mathrm{cm}^{3}\right]$} \\
\hline
\end{tabular}

Table 5: reactive viscosity parameter Hercorub EPDM $005 \mathrm{~K}$

\begin{tabular}{lrl}
\hline Parameter & Value & Unit \\
\hline $\mathrm{n}$ & 0.493 & {[]} \\
$\tau^{*}$ & 43500 & {$[\mathrm{~Pa}]$} \\
$\mathrm{B}$ & 2.203 & {$[\mathrm{~Pa} . \mathrm{s}]$} \\
$\mathrm{Tb}$ & 3845.3 & {$[\mathrm{~K}]$} \\
$\mathrm{C} 1$ & 1 & {[]} \\
$\mathrm{C} 2$ & 0 & {[]} \\
\hline
\end{tabular}


To investigate the influence of mold temperature on interface temperature, multiple simulations were performed. As boundary conditions, the water temperature for the rubber cavity is set at $180^{\circ} \mathrm{C}$, and the temperature of the water at the side of the thermoplastic product is varied in steps of $10^{\circ} \mathrm{C}$ between $40^{\circ} \mathrm{C}$ and $80^{\circ} \mathrm{C}$ resulting in 5 temperature setting. The rubber material is injected at $80^{\circ} \mathrm{C}$ in 0.5 seconds. In further discussion the location 0 represents the interface of the product, negative values correspond to a location in thermoplastic part, while positive values determine a location in the thermoset product.

\subsection{Simulation results}

\subsubsection{Thermal results}

By performing Cool (FEM) Fill Pack simulations, the temperature distribution in the mold and across the product can be determined for different temperature settings. Figure 4, left shows the temperature distribution across the plate product for the top, middle and bottom side of the product for the 180-60 setting immediately after the injection phase. This nomenclature complies with a thermoset water temperature of $180^{\circ} \mathrm{C}$ and a thermoplastic water temperature of $60^{\circ} \mathrm{C}$. The temperature distribution is governed by the interaction between mold and material. As shown in this figure, the temperature does not only vary as a function of the location, but also in the through-thickness direction. There is a clear temperature difference between the top and bottom side of product. A detailed view of this variation of temperature is shown in Figure 4 (right-hand side) and is measured to be around $20^{\circ} \mathrm{C}$. This temperature distribution was not intended and is caused by the mold construction, which was established before having the extended knowhow of the influence of temperature on the adhesive strength Therefore the thermal control of the mold was not optimized for the present study.
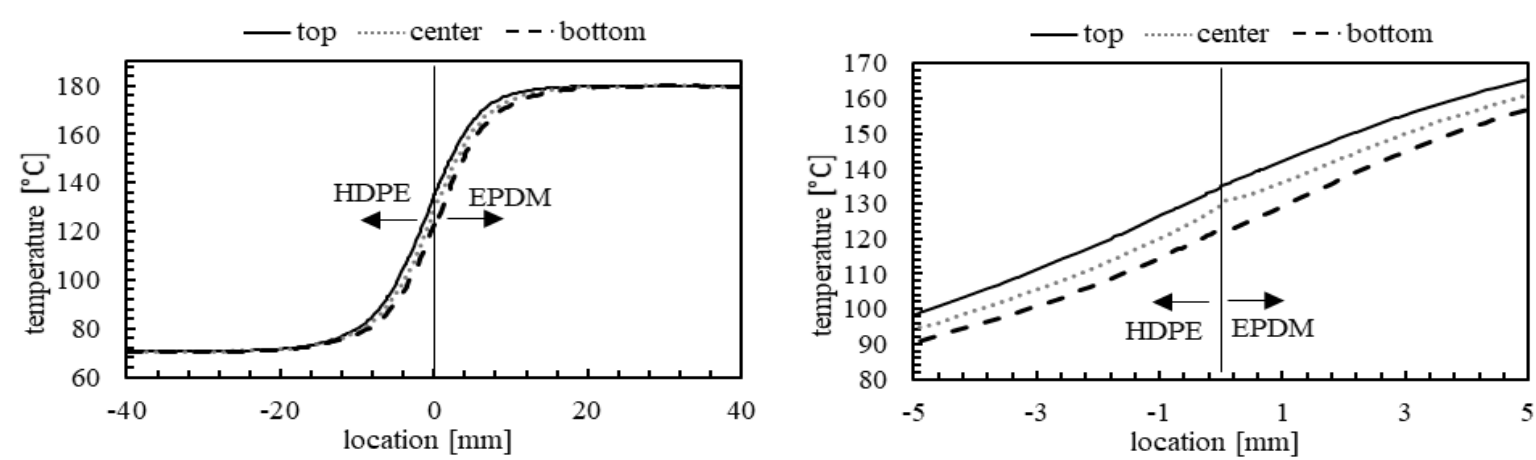

Figure 4: Simulated top, middle and bottom temperature across the product for the 180-60 setting at the end of the injection phase. (left), close up of the interface top, middle and bottom temperature (right),

The resulting temperature distribution in the through-thickness direction at the end of the injection phase is shown in Figure 5 left. As stated before, this temperature is not constant due to the mold construction. As a result of the variation of temperature, the resulting degree of melting will also vary throughout the thickness. The difference between bottom and top temperature is similar for all temperature settings. By using the exponential fit, given in Figure 3, the interface temperature can be used to calculate the degree of melting $\left(\mathrm{D}_{\mathrm{m}}\right)$ for all these temperature settings. The degree of melting for these given temperature distributions are shown in Figure 5 right. The resulting degree of melting ranges from 0 to 0.2 in the lowest temperature setting (180-40) and increases in the highest temperature setting (180-80) from 0.2 to 

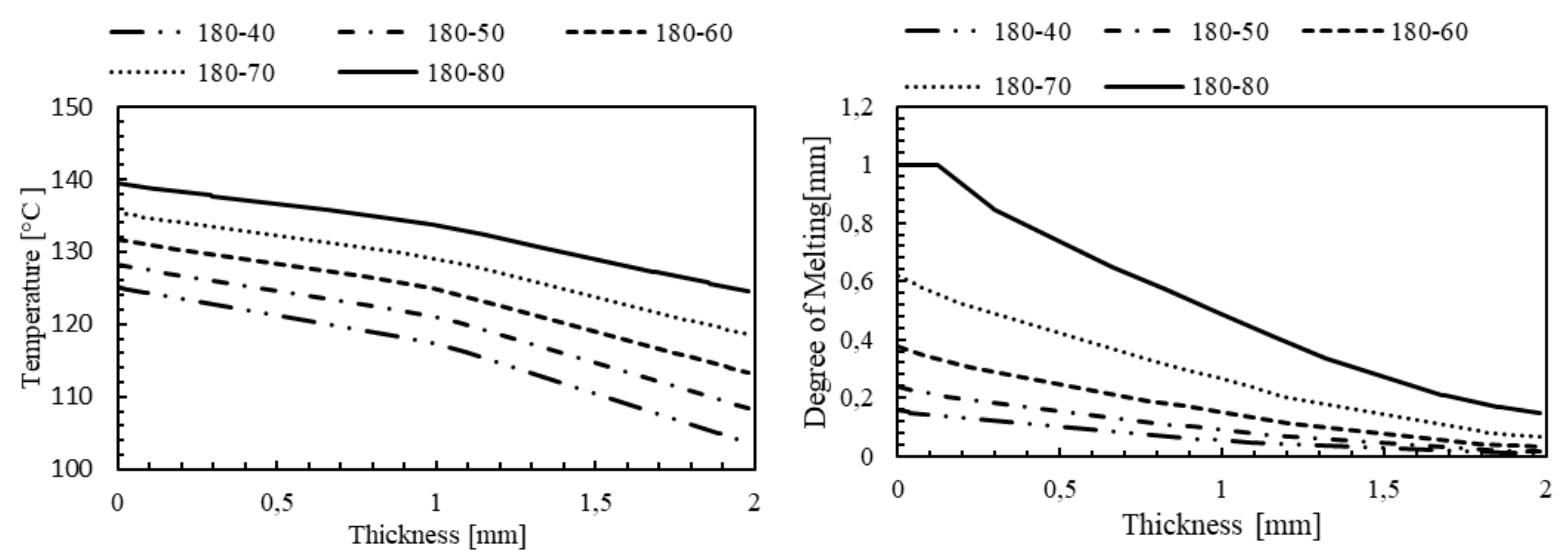

Figure 5. left: Temperature distribution through thickness at the interface of the plate product for 5 temperature settings. Right: Degree of melting of HDPE M80064SE through thickness for various temperature settings.

1. The difference between bottom and top increases due to the exponential behavior of the melting curve. Having interface melting of 1 should be avoided to reduce interface deformation and to avoid differential thermal contractions and interface deformation. These deformation and contraction will result in defective parts.

\subsubsection{Curing results}

The second important simulation result is the degree of cure of the thermoset rubber. The curing of the thermoset rubber starts as soon as the material is injected into the cavity of the mold. Curing is modeled as a function of time and location and is governed by the actual temperature in the cavity and time as stated by the Kamal model. As shown in the previous section, the temperature distribution across the cavity and through thickness is not constant, which will influence the local curing speed and final degree of cure. Due to the low interface temperature, compared to the optimal curing temperature, full curing at the interface will take longer compared the bulk part of the thermoset rubber. To avoid cohesive failure, a high interface curing is needed. The simulation of the degree of cure as a function of the location across the thermoset part is shown in Figure 6. Although curing is above $90 \%$ for most of the part at $341 \mathrm{~s}$, curing at the interface is still below $10 \%$ and will result in a very low rubber strength and a low cohesive strength. Simulations predict that an extra 1000 seconds are needed to reach $75 \%$ curing at the interfaces for Hercorub EPDM 005K.

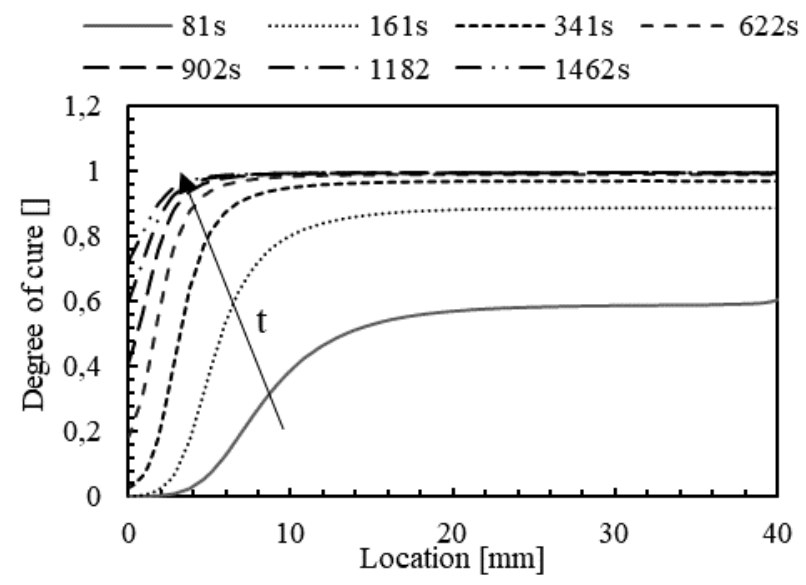

Figure 6: Degree of cure of Hercorub EPDM 005K as a function of time and location in the 180-60 temperature setting. 
The degree of cure can be used to calculate the final rubber strength and thus cohesive strength during the curing phase.

\subsection{Test results.}

By combining the rubber strength and the adhesive strength, it is possible to examine when the rubber strength will exceed the adhesive strength, dependent on the degree of melting of the thermoplastic material.

Figure 7 shows the combination of both expected adhesive and cohesive strength of the interface for the temperature setting of 180-60. As stated before, cohesive strength increases as a function of curing time, shown in this figure for 7 different time steps. The lines defining this strength are inclining as temperature is not constant in the through-thickness direction of the product, shown in Figure 5 (left). The thick, curved line in this figure defines the expected adhesive strength between the Sabic HDPE M80064SE and the Hercorub EPDM 005K. This adhesive strength also varies from 0.35MPa to 7.9MPa, and crosses the thin lines defining the cohesive strength. One could state that as long as the healing curve crosses the lines defining the rubber strength, a mixed failure mode (cohesive and adhesive) should occur. This seems to be valid in the given case with a curing time of 1462s, shown in Figure 7 (right, A). The interface of this sample has failed in a mixed manner. Only a small portion of the rubber (black zones) are on the left side the fracture surface, showing where the rubber has failed. Regions without rubber have failed in an adhesive manner. Further curing should reduce the amount of cohesive failing. For the second case at a curing time of 902s, shown the Figure 7 (right, B). The situation shows a complete cohesive failure for one sample and a mixed for another sample. However, comparing the data for the $1462 \mathrm{~s}$ and the $902 \mathrm{~s}$ both should have a mixed failure. The reason for this discrepancy is thought to be a more complex failure behavior. At first, an adhesive failure is expected to happen in the lower temperature region. This increases the tension in the remaining cross section, above the rubber strength, but under the adhesive failure strength, creating the possibility of a complete rubber failure.

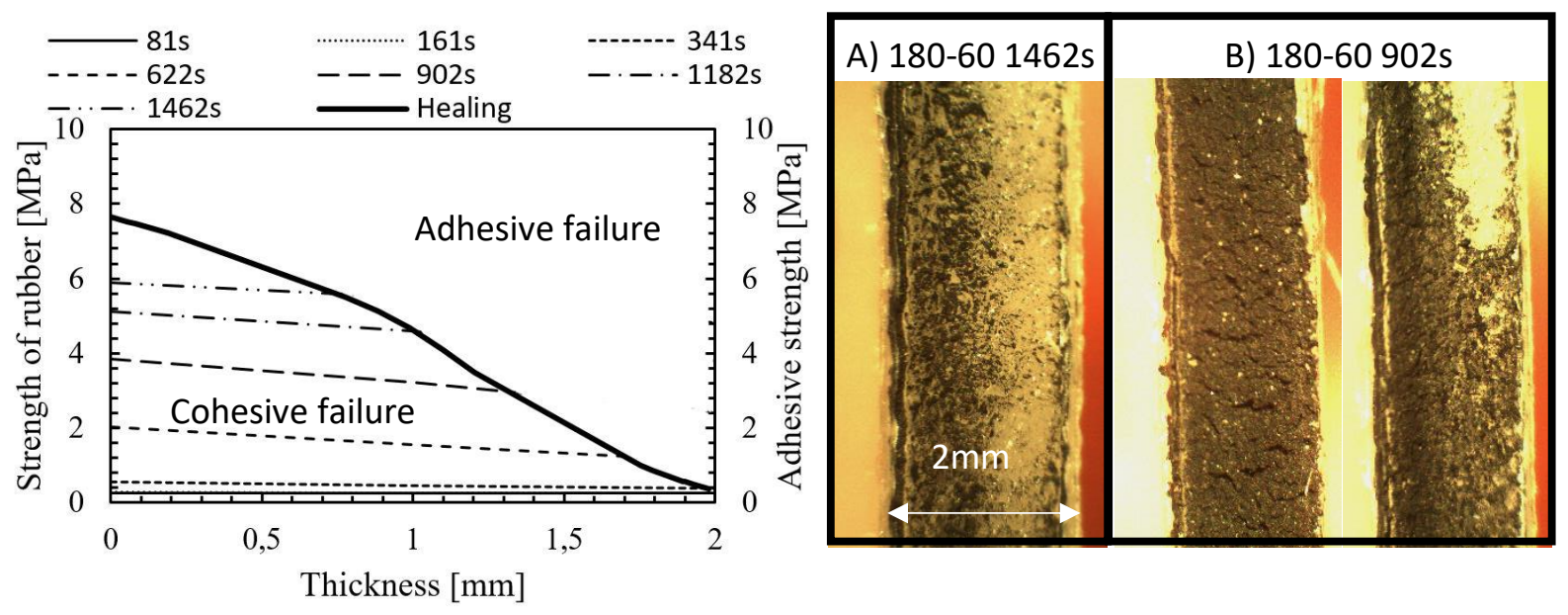

Figure 7: Temperature setting 180-60. Left: simulated strength of Hercorub EPDM 005K at the interface for increasing curing times (thin lines, left scale bar) and adhesive strength of interface due to healing for HDPE M80064SE (thick line, right scale bar), Right: State of interface of three samples after testing for two curing times. 1 sample for 1462s (A) and two samples for $902 s(B)$.

The measured interface strength is shown in Table 6. From these results it can be concluded that initial increasing of curing time strongly increases the interfacial strength. However, as expected if the rubber strength reaches the adhesive strength, increasing curing time does not increase the interface strength much further as adhesive strength becomes the limiting factor, also concluded in Figure 7. The presented table also shows the simulated interface strength determined via the presented method. 


\begin{tabular}{rrrr}
\hline $\begin{array}{c}\text { Curing } \\
\text { time }\end{array}$ & $\begin{array}{r}\text { Average } \\
\text { interface } \\
\text { strength }\end{array}$ & Std & $\begin{array}{r}\text { Simulated } \\
\text { interface } \\
\text { strength } \\
{[\text { [MPa }]}\end{array}$ \\
\hline s] & 1.74 & 0.034 & 1.41 \\
622 & 3.28 & 0.478 & 2.70 \\
902 & 3.61 & 0.273 & 3.61 \\
1182 & 4.25 & 0.347 & 4.10 \\
1462 & & & \\
\hline
\end{tabular}

5. Interface strength prediction

Via scripting, and after performing the injection molding simulations, the results in section 4 can be used to create additional simulation plots showing the expected interfacial strength on the complete product. The used script is based on the available Autodesk Moldflow results. The following two results from the Cool(FEM), Fill-Pack analysis, are used: 'Temperature, part insert' and 'Conversion at node'. The first result shows the temperature of the thermoplastic insert as a function of time. The 'Conversion at node' shows the local degree of cure as a function of time The method is shown in a flowchart, presented in Figure 8.

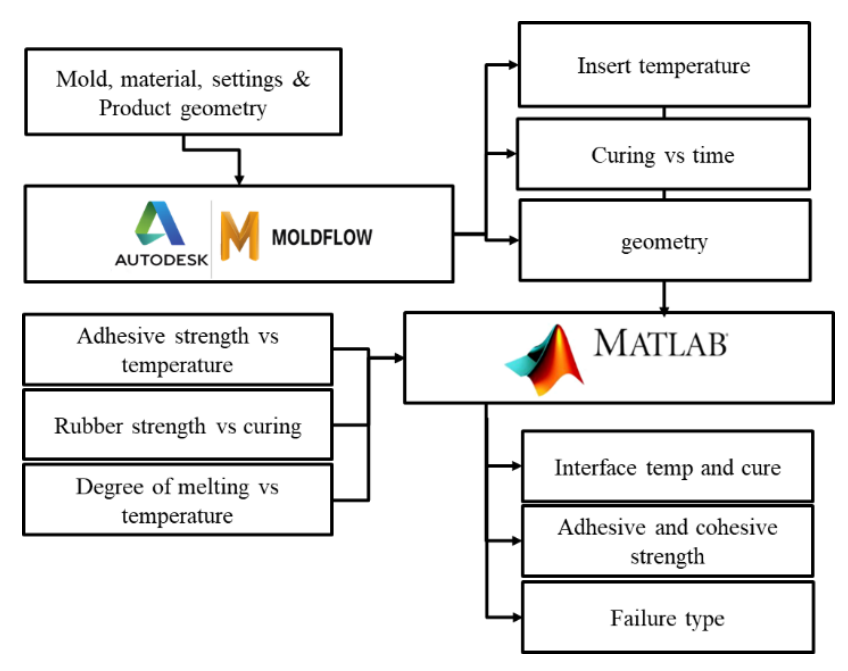

Figure 8: Flowchart: method to define the interfacial strength.

The first result given by the script, applied on the interface of the complete plate product, is the expected adhesive strength of the complete interface, combined with the interface temperature. This is shown in Figure 9. This figure shows the expected adhesive strength for the 150x $2 \mathrm{~mm}$ (cfr. Figure 1, right) interface in case of the temperature setting 180-60. In this figure, the complete interface temperature, at the end of the injection phase is shown in row ' $A$ '. It is clear that this interface temperature varies throughout the thickness and as a function of the position on the interface. Via the equation for $\sigma_{\mathrm{Dm}}$, shown in Figure 3, the local adhesive strength across the complete interface can be calculated and visualised (row 'B' of Figure 9). Via the same method, the degree of cure on the interface of the thermoset rubber also can be visualised as a function of time. Figure 10 shows the results for two time steps for the 180-60 situation, time steps $902 \mathrm{~s}$ and $1462 \mathrm{~s}$. Row 'A' in Figure 10 shows the resulting degree of cure at the complete interface for the two given time steps. The tested interfaces (cfr. Figure 3, right and results in Table 6) are located in the middle of the product, defined by the square marker on the figures. The local degree of cure can be transformed into a local cohesive strength, this is shown in row ' $\mathrm{B}$ '. Row ' $\mathrm{C}$ ' compares the adhesive and cohesive strength and shows which one is the lowest, and thus defines the failure type in tensile testing. Row ' $\mathrm{D}$ ' shows the local interface strength result by applying equation 6 . A remarkable 
resemblance can be found by comparing row ' $\mathrm{C}$ ' with the results in Figure 7, right. for the time step of 902s. An almost complete cohesive failure is expected and found. For the time step of 1462s, a mixed failure is expected as discussed in section 4.3 and also found experimentally. These results show the possibilities of the presented method which can be used in a next step to optimise the interfacial strength. The method shows promising results and will be further tested for other material combinations.

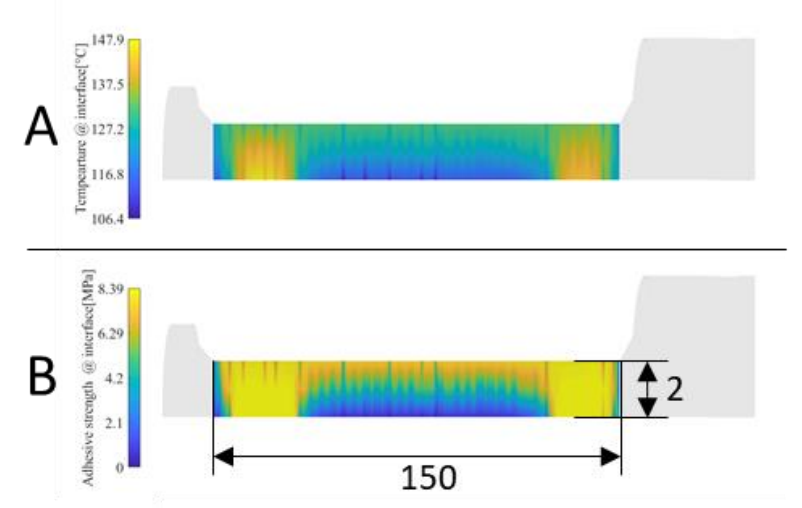

Figure 9: Interface temperature (a) and expected adhesive strength (b)

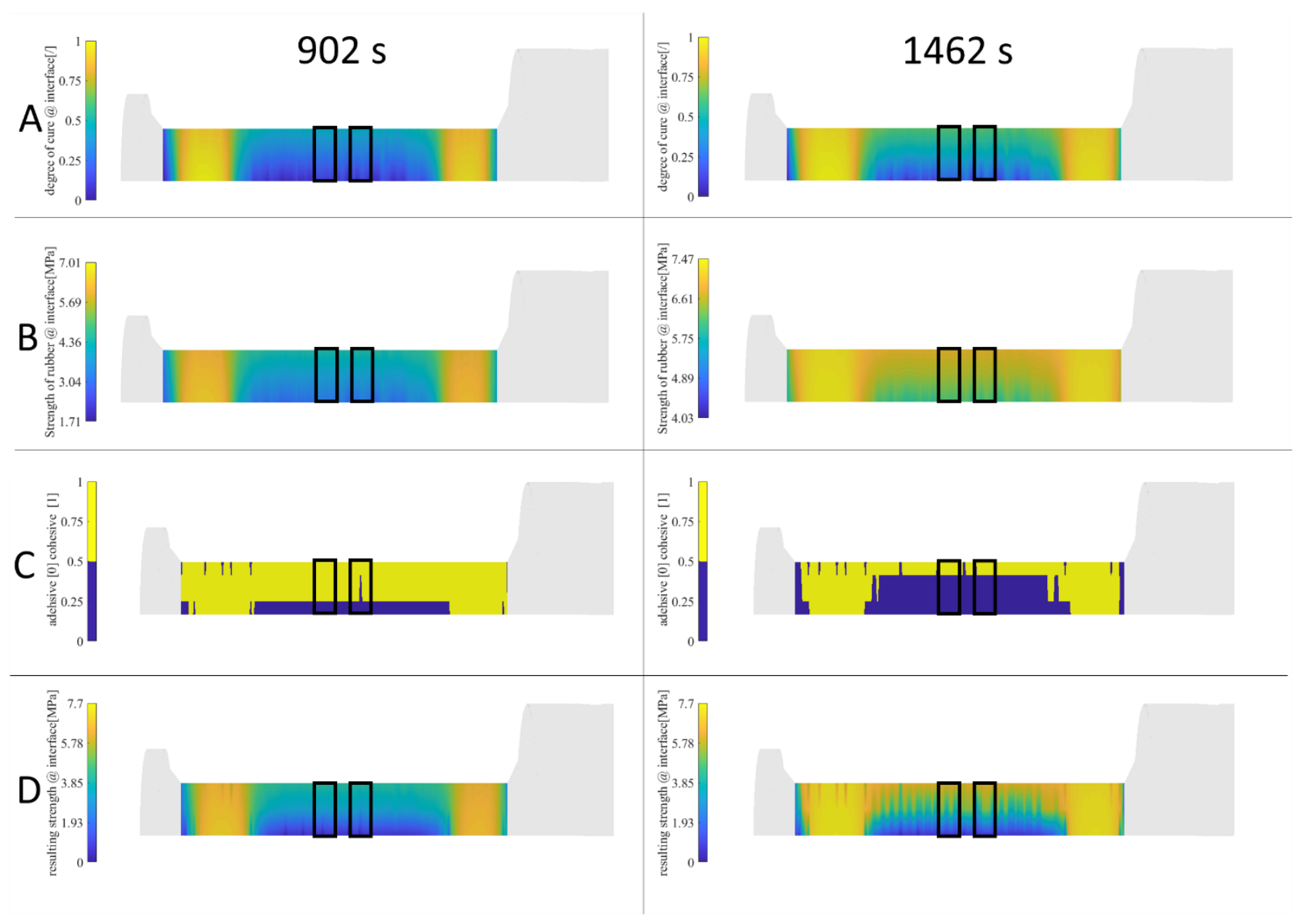

Figure 10:Interface conditions for two curing times. Left, 902s, Right 1462s. row A, shows the local interface cure for the complete product. Row B shows the resulting cohesive strength. Row $C$ shows an estimate of failure mode (adhesive or cohesive). Row $D$ shows the resulting, local interface strength.

\section{Conclusions}


In this work, a method has been presented in order to predict the interface strength between a compatible semi-crystalline thermoplastic material and a thermoset rubber. For this, knowledge on the healing of interfaces in thermoplastic materials was used which is often used in thermoplastic composite production. This thermoplastic healing model has successfully been adapted to function in a the thermoset overmolding case. The healing model was combined with the knowledge about the strength of rubbers as a function of curing degree. The combination of those two models has resulted into a novel method to predict the interfacial strength of the given material combination. At the given moment, the method is only useful for unidirectional loading conditions. However, the developed numerical model requires only a limited number of experimental tests to define specific input data and so can easily be extended towards other load cases. The accuracy of the model is mainly dependent on the quality of the input data for adhesive and cohesive strength and the accuracy of the injection moulding simulations. The presented method was used to create a script which combines the developed knowledge and creates a new type of result plot showing the influence of the processing conditions on the final properties of the interface. As a follow-up, this data will be used to write an interface towards structural software order to perform 'as produced' product simulations.

7. Acknowledgements.

The authors acknowledge the KU Leuven Impulsfonds for the financial support through project IMP/14/043 and the Company Hercorub NV for providing the uncured rubber material. G.J. Bex acknowledges the Flanders Research Foundation (FWO) for his PhD grant strategic basic research.

References

[1] Kamal, Isayev, and Liu, Injection Molding; Technology and Fundamentals. Munich: Hanser, 2009.

[2] D. Weng, J. Adries, P. Morin, K. Saunders, and J. Politis, "Fundamentals and material development for thermoplastic elastomer (TPE) overmolding," J. Inject. Molding Technol., vol. 4, no. 1, pp. 22-28, 2000.

[3] A. R. Carella, C. Alonso, J. C. Merino, and J. M. Pastor, "Sequential injection molding of thermoplastic polymers. Analysis of processing parameters for optimal bonding conditions," Polym. Eng. Sci., vol. 42, no. 11, pp. 2172-2181, 2002.

[4] D. Y. Huang and R. S. Chen, "Bonding strength at solid-melt interface for polystyrene in a sequential twostaged injection molding process," Polym. Eng. Sci., vol. 39, no. 11, pp. 2159-2171, 1999.

[5] R. M. Gouker, S. K. Gupta, H. A. Bruck, and T. Holzschuh, "Manufacturing of multi-material compliant mechanisms using multi-material molding," Int. J. Adv. Manuf. Technol., vol. 30, no. 11-12, pp. 1049$1075,2006$.

[6] H. R. Brown, “The Adhesion Between Polymers,” Annu. Rev. Mater. Sci., vol. 21, no. 1, pp. 463-489, 1991.

[7] A. Islam, H. N. Hansen, and M. Bondo, "Experimental investigation of the factors influencing the polymer-polymer bond strength during two-component injection moulding," Int. J. Adv. Manuf. Technol., vol. 50, no. 1-4, pp. 101-111, 2010.

[8] J. Crank and G. S. Park, Diffusion Polymers, vol. 20. 1996.

[9] S. Prager and M. Tirrell, "The healing process at polymer-polymer interfaces," J. Chem. Phys., vol. 75, no. 10, pp. 5194-5198, 1981.

[10] S. Guo, A. Ait-kadi, and M. Bousmina, "A modified model predictions and experimental results of weldline strength in injection molded PS / PMMA blends," Polymer (Guildf)., vol. 45, pp. 2911-2920, 2004.

[11] M. B. Bouwman, T. Donderwinkel, E. Krämer, S. Wijskamp, and F. Costa, "Overmolding - An integrated design approach for dimensional accuracy and strength of structural parts," CAMX 2016 - Compos. Adv. Mater. Expo, 2016.

[12] F. L. (evonik Industries), "High-Performance Polymers in Plastic-Rubber Composites," Composites. Evonik industries.

[13] G. Bex, F. Desplentere, J. De Keyzer, and A. Van Bael, "Two-component injection moulding of thermoset 
rubber in combination with thermoplastics by thermally separated mould cavities and rapid heat cycling," Int. J. Adv. Manuf. Technol., 2017.

[14] G. Bex, W. Six, B. Laing, J. De Keyzer, F. Desplentere, and A. Van Bael, "Effect of process parameters on the adhesion strength in two-component injection molding of thermoset rubbers and thermoplastics," vol. 46495, pp. 1-9, 2018.

[15] J. Tierney and J. W. Gillespie, "Modeling of in Situ strength development for the thermoplastic composite tow placement process," J. Compos. Mater., vol. 40, no. 16, pp. 1487-1506, 2006.

[16] R. P. Wool, "Polymer Entanglements," Macromolecules, vol. 26, no. 7, pp. 1564-1569, 1993.

[17] F. Yang and R. Pitchumani, "A kinetics model for interphase formation in thermosetting matrix composites," J. Appl. Polym. Sci., vol. 89, no. 12, pp. 3220-3236, 2003.

[18] C. Fetecau, F. Stan, and D. Dobrea, "Investigation of the adhesion interface obtained through twocomponent injection molding," AIP Conf. Proc., vol. 1315, pp. 701-706, 2010.

[19] C. Fetecau, D. V. Dobrea, and I. Postolache, "Overmolding Injection Molding Simulation Of Tensile Test Specimen," Int. J. Mod. Manuf. Technol., vol. II, no. 2, 2010.

[20] W. Six, F. Desplentere, and A. Van Bael, "Simulation and optimization of vulcanization in the adhesion area in a 2K Thermoset-Thermoplastic product," in Polymer \& mould innovations, 2016, pp. 1-5.

[21] M. R. Kamal and S. Sourour, "Kinetics and Thermal Characterization of Thermoset Cure," Polym. Eng. Sci., vol. 13, no. 1, pp. 59-64, 1973.

[22] G. Milani, F. Milani, and P. Leonardo, "Simple Kinetic Numerical Model Based on Rheometer Data for Ethylene - Propylene - Diene Monomer Accelerated Sulfur Crosslinking,” vol. 1, no. 1, 2011.

[23] A. I. Isayev and M. Wan, "Injection Molding of a Natural Rubber Compound: Simulation and Experimental Studies," Rubber Chem. Technol., vol. 71, no. 5, pp. 1059-1072, 1998.

[24] N. P. Cheremisinoff and D. Ph, Condensed encyclopedia of polymer engineering terms, vol. 39, no. 03. 2013.

[25] G. Ramorino et al., "Injection molding of engineering rubber components: A comparison between experimental results and numerical simulation University of Brescia ( Italy ) - Department of Mechanical and Industrial Engineering," vol. 3, pp. 551-554, 2010.

[26] Kroll, Andreas (2000). Grey-box models: Concepts and application. In: New Frontiers in Computational Intelligence and its Applications, vol.57 of Frontiers in artificial intelligence and applications, pp. 42-51. IOS Press, Amsterdam 